\title{
Three-dimensional microstructure and numerical calculation of elastic properties of alpine snow with a focus on weak layers
}

\author{
Berna KÖCHLE, Martin SCHNEEBELI \\ WSL Institute for Snow and Avalanche Research SLF, Davos Dorf, Switzerland \\ E-mail: schneebeli@slf.ch
}

\begin{abstract}
The microstructure and stratigraphy of a snowpack determine its physical behaviour. Weak layers or weak interfaces buried under a slab are prerequisites for the formation of dry-snow slab avalanches, and a precise characterization of weak layers or interfaces is essential to assess stability. Yet their exact geometry and micromechanical properties are poorly known. We cast weak layers and their adjacent layers in the field during two winters and reconstructed their three-dimensional microstructure using X-ray microcomputer tomography. The high resolution of $10-20 \mu \mathrm{m}$ allowed us to study snow stratigraphy at the microstructural scale. We quantified the microstructural variability for 32 centimetre-sized layered samples and we calculated Young's modulus and Poisson's ratio by tomography-based finite-element simulations. Layers in a sample could therefore be differentiated not only by a change in morphology or microstructure, but also by a change in mechanical properties. We found a logarithmic correlation of Young's modulus with density for two different density ranges, consistent with previous studies. By calculating the relative microstructural changes within our samples, we showed that a large change could indicate a potential weak layer, but only when the weak layer and both adjacent layers, i.e. the sandwich, were considered.
\end{abstract}

KEYWORDS: avalanches, snow, snow mechanics, snow microstructure, snow physics

\section{INTRODUCTION}

Layers in a snowpack are differentiated by their morphological and physical properties, both of which depend on snow microstructure. The stratigraphy, and thus the differences in microstructure, also determine the mechanical behaviour of a snowpack. Studying layering at the microstructural scale $\left(10^{-2}-10^{-4} \mathrm{~m}\right)$ is essential to find microstructure-based parameters that reveal layers relevant to mechanics (e.g. Kry, 1975).

Snow is constantly deforming due to its high homologous temperature, and the deformation behaviour is highly strainrate dependent (Narita, 1980; Scapozza, 2004). Creep compacts the snow permanently, but does not lead to its failure. In contrast, fast deformation induces high strain rates $\left(>10^{-2} \mathrm{~s}^{-1}\right)$, whereby snow deforms mainly elastically and can fracture and fail in a brittle way. Weak layers consist of snow structures that are more prone to brittle fracture and failure than others. If buried under a bonded slab, an evolving and propagating crack therein can lead to partial or entire failure of a snowpack. The formation of dry-snow slab avalanches is mainly a brittle process, caused by the failure of a weak layer (Schweizer and others, 2003). The identification of potential weak layers is a key element in assessing the stability of a snowpack. To distinguish weak layers from non-weak layers, microstructural differences act as an indicator (e.g. Schweizer and Wiesinger, 2001). Moreover, in many cases, the detection of weak layers requires mechanical stability tests, whereby several weak layers can be found. Their identification and characterization by these field tests can be difficult for manifold reasons. First, the most important drawback is the limited spatial resolution of a traditional snow profile. Second, weak layers are often very thin, making it difficult to precisely localize the failure in the snow profile. Third, structural and mechanical properties are hard to quantify for layers $<1 \mathrm{~cm}$ thick. For these reasons weak layer properties are seldom quantified or quantifiable by hand measurements in the field.

Highly resolved measurements of the mechanical penetration resistance (e.g. with the SnowMicroPen (SMP); Schneebeli and others, 1999) enable us to overcome the limited resolution of hand measurements. Although the force/signal interpretation is not clear (Johnson and Schneebeli, 1999; Marshall and Johnson, 2009; Löwe and Van Herwijnen, 2012), microstructural parameters can be deduced with high resolution. The SMP was recently used to quantify relative differences between layers (Lutz and others, 2009; Van Herwijnen and others, 2009) and to detect weak layers by signal analysis (Pielmeier and Schweizer, 2007; Bellaire and others, 2009; Van Herwijnen and others, 2009). However, uncertainty remains in the signal interpretation with respect to weak layers. One key factor is that the microstructure of weak layers is poorly known.

Microstructural layering was also studied in this context, by imaging cast snow structures with surface sections. Kronholm and others (2004) and Pielmeier and Schweizer (2007) compared them qualitatively with SMP measurements. Föhn and others (1998) used this method to quantify the microstructure of an embedded weak layer and to relate it to measured shear strength. They concluded that stereological measurements cannot differentiate the weak layers from the adjacent layers well enough to find such a relation. They suggested that three-dimensional (3-D) reconstructions are necessary to describe a mechanically relevant change in microstructure by microstructural parameters alone. In the context of investigating weak layer properties, this has not yet been done.

The two elastic constants, Young's modulus $E$ and Poisson's ratio $\nu$, are characteristic material properties of 
snow and are required in many applications, for example in fracture mechanical models of slab avalanche release (McClung, 1981; Heierli and others, 2008), but they are difficult to obtain experimentally. The mechanical testing of fragile snow types is very challenging, even in a wellcontrolled laboratory environment. Studies attempting a correlation between $E, \nu$ and microstructure were thus often limited to less fragile and often dense snow. Until now most correlations have been based on density, as high-resolution microstructural measurements are not available. To apply such correlations, a reliable density measurement is required, i.e. sufficiently resolved to ensure the sample or layer homogeneity. This requirement often hinders the use of density-based correlations for most of the weak layers: if very thin, density cannot easily be measured by hand. Furthermore, most snow types show anisotropic physical behaviour to a certain degree (Löwe and others, 2013), i.e. from isotropic to transverse isotropic in the horizontal plane (Srivastava and others, 2010), and hence have different Young's moduli depending on the direction. The elasticity tensor $C_{i j k l}$ relating stress and strain is required for a complete characterization of snow in terms of elasticity and to calculate the Young's moduli in each direction, but $C_{i j k l}$ of a given snow sample cannot be measured experimentally; a snow sample can only be tested once, since most mechanical tests are destructive. Consequently, experimentally determined Young's moduli are mostly measured in the vertical direction and assume an isotropic material.

Numerical experiments, in contrast, allow us to calculate the full elasticity tensor based on real non-idealized 3-D snow structure. X-ray microcomputer tomography $(\mu-\mathrm{CT}$; Coléou and others, 2001; Schneebeli, 2002) measures objective and highly resolved snow microstructure, which can be used as geometric input. This method was used for elastic simulations to study stress distribution (Pieritz and others, 2004), to study the evolution of elastic properties during temperature-gradient metamorphism experiments (Schneebeli, 2004; Srivastava and others, 2010) and for viscoelastic simulations to study creep (Theile and others, 2011).

In this study we investigate snow stratigraphy based on $\mu$-CT measurements to quantify the microstructural change. We cast natural layered snow samples in the field with a focus on embedded weak layers. Our 32 samples cover a wide range of snow types and densities from 100 to $500 \mathrm{~kg} \mathrm{~m}^{-3}$. The microstructure of these centimetre-sized samples was reconstructed using $\mu-\mathrm{CT}$, as described by Heggli and others (2011). The 3-D data constituted the geometric input for elastic finite-element (FE) simulations (Van Rietbergen and others, 1995). The calculated elastic constants, Young's modulus and Poisson's ratio were used together with microstructural information to identify layers and quantify their structural differences. We also found a logarithmic correlation for the Young's modulus with density, based on our FE simulations. Weak layers were identified using mechanical field tests, and the corresponding layers in the $\mu$-CT data were determined, in order to elucidate the differences between weak layers and non-weak layers. We found that the relative microstructural changes between a weak layer and the adjacent layers are up to ten times larger than between adjacent layers in general. The magnitude of relative change between layers is an indicator of a potential weak layer, but only if both the layer above and the layer below are considered.

\section{METHODS}

\section{Data acquisition of layered snow}

During winter 2008/09 and 2009/10 seasonal snow, primarily weak layers and their adjacent layers, was sampled in the field near Davos, Switzerland. Winter 2008/09 had average conditions regarding snowfall events and snow-cover stability. Winter 2009/10 had temperatures and snow heights below the long-term mean and was characterized by mostly unstable snowpack conditions. Samples were taken at altitudes in the range 1600 $2600 \mathrm{~m}$ a.s.I., on slopes of all aspects and angles between 0 and $45^{\circ}$. The weak layers were determined by hand profiles and stability tests using extended compression and rutschblock tests, as described by Winkler and Schweizer (2009). Samples of $5 \mathrm{~cm} \times 5 \mathrm{~cm} \times 7 \mathrm{~cm}$ were cut parallel to the layering. For safe transport from the field to the cold laboratory, each sample was carefully cast by replacing the air in the sampled snow with dyed diethyl-phthalate and then freezing it with dry ice. In the cold laboratory, the cast samples were processed and scanned nondestructively with an X-ray microcomputer tomograph (SCANCO Medical AG, $\mu$-CT40) with a nominal resolution of $10 \mu \mathrm{m}$ voxel size. As described by Heggli and others (2011), each sample was scanned before and after ice sublimation, necessary for a $\mu-\mathrm{CT}$ analysis of cast snow to detect air bubbles enclosed during the casting process. The inverse of the segmented air matrix gives the ice matrix, and the original snow microstructure was then reconstructed by additional image processing. (More details are given by Heggli and others, 2011.) The 32 digitized layered samples used in this study had a sample height of $4.5-7.0 \mathrm{~cm}$ and a side length of up to $1.5 \mathrm{~cm}$.

\section{Microstructure-based identification of layers in $\mu-C T$ samples}

Microstructural layering was analysed by calculating the vertical variation in geometry. The following parameters were calculated within overlapping horizontal slices of $2 \mathrm{~mm}$ thickness over the entire sample height from the 3-D data: density $(\rho)$, specific surface area (SSA), the number of ice structures per millimetre (trabecular number, Tb.N), ice thickness (Th), pore thickness (Sp) and the connectivity density (Conn.D). Snow density was obtained by calculating the ice volume fraction, $\phi_{\text {ice, }}$ by counting voxels and multiplying $\phi_{\text {ice }}$ by the density of ice, $\rho_{\text {ice }}=917 \mathrm{~kg} \mathrm{~m}^{-3}$. With the triangulation of the ice surface, the ice surface area was calculated and then divided by the ice volume to obtain the SSA. Tb.N, Th and Sp were computed by the method based on the inscription of spheres (Hildebrand and Rüesegger, 1997). Conn.D was calculated as described by Odgaard and Gundersen (1993). Based on the resulting vertical distribution of these parameters and an examination of the reconstructed sample by eye, individual layers were designated. Cubic subvolumes were cut from these layers, as shown in Figure 1. These subvolumes were finally used to numerically calculate elastic properties. The snow within these layers was morphologically classified according to the scheme of Fierz and others (2009).

When two snow types were present in equal amounts (e.g. faceted crystals (FC) and rounded grains (RG)), the snow in this layer was classified as a mixture (e.g. FC/RG). An additional designation of layers in the $\mu$-CT sample (weak layer, layer above the weak layer, and layer below the 


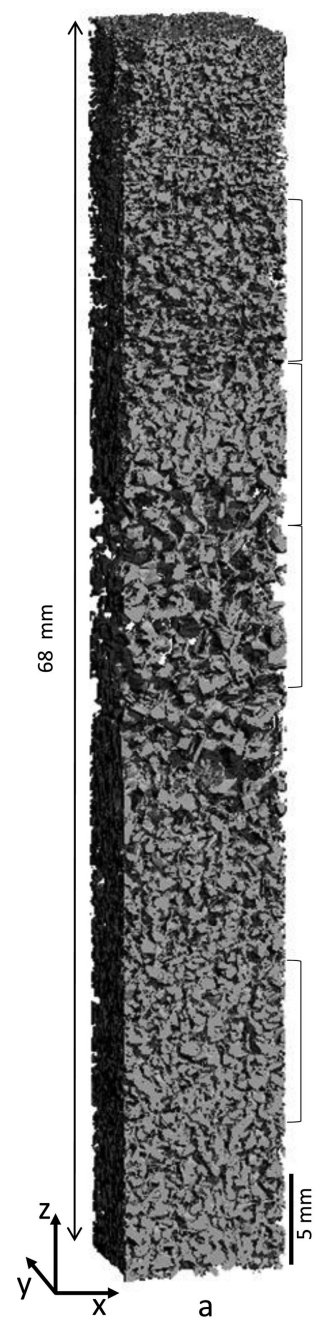

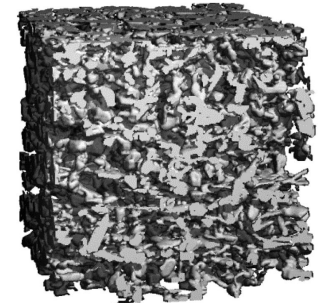
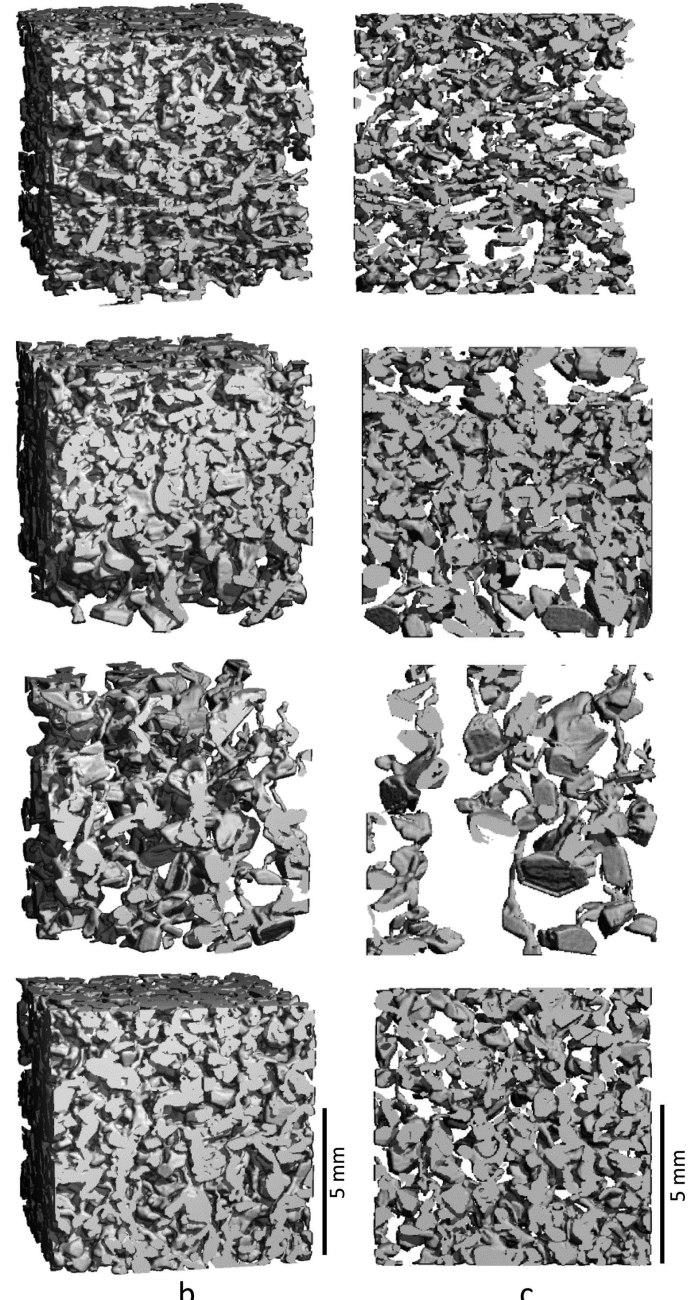

Fig. 1. Three-dimensional reconstruction from $\mu$-CT measurements of a natural, layered snow sample. (a) Low-density cups forming a weak layer (in the middle of the sample) below a thin melt/freeze crust, both buried under small rounded grains (sample name WG3; volume $8.9 \mathrm{~mm} \times 8.9 \mathrm{~mm} \times 68 \mathrm{~mm}$; resolution $0.01 \mathrm{~mm}$ ). (b) Cubic subvolumes cut from (a) for FE calculations with side length $8.91 \mathrm{~mm}$ (resolution $0.03 \mathrm{~mm}$ ). (c) Vertical cross sections in the $x-z$ plane of $1.5 \mathrm{~mm}$ horizontal thickness.

weak layer) was made according to observations in the field. Allocating field observations to the highly resolved 3-D data was often difficult, and in ambiguous cases the layers were not classified as layer above, weak layer or layer below.

\section{Finite-element calculations}

A voxel-based FE code (Van Rietbergen and others, 1995, 1996) was used to calculate $E$ and $\nu$. For each of the 104 cubic subvolumes, one linear-elastic FE calculation simulating uniaxial strain in the vertical direction was made with displacement boundary conditions. Nodes located at the bottom, $Z_{\mathrm{min}}$, had zero displacement, and nodes on the top, $Z_{\max }$, had a prescribed vertical displacement corresponding to unit strain. Nodal displacement at lateral surfaces was restricted to the $z$-direction. The geometry of the snow structure was implemented by a voxel-to-element conversion of the segmented subvolume, such that, once unconnected ice structures had been removed, each ice voxel was converted into an eight-node brick element. Air voxels were not considered in the simulation. All elements were homogeneous, isotropic, of the same size, shape and orientation, and were assigned to the corresponding material property of ice. For ice, isotropic, linear-elastic behaviour was assumed, with $E=10 \mathrm{GPa}$ and $\nu=0.3$ (Petrovic, 2003).

\section{Representative volume element}

The minimum size required to capture the representative bulk property with respect to $E$ was determined by calculating $E$ of cubic subvolumes of increasing size within a given volume. A variation of $E$ of less than $\pm 20 \%$ determined the size of the representative volume element (RVE). The size of such a RVE was determined exemplarily for three different snow types: rounded snow (RG), faceted snow (FC) and depth hoar (DH), all with densities $<300 \mathrm{~kg} \mathrm{~m}^{-3}$. We assume that the snow in our given volume can be considered structurally isotropic. Nevertheless, to account for possible structural variability, the RVE calculations were started from four different corners and the cube side length was increased stepwise in all three directions until the entire volume was filled. To reduce computational time, the resolution of the 3-D data was reduced by a factor of three or four (for snow of large grain size), maintaining porosity and specific surface area.

\section{Calculation of Young's modulus and Poisson's ratio}

Assuming a linear-elastic and homogeneous material, the relation between stress, $\sigma$, and strain, $\epsilon$, is given by the generalized Hooke's law

$$
\sigma_{i j}=C_{i j k l} \epsilon_{k l}
$$


written in index notation, where $\sigma_{i j}$ and $\epsilon_{k l}$ are second-order tensors and $C_{i j k l}$ is a fourth-order tensor and referred to as the elasticity tensor. If the material is considered to be isotropic, Hooke's law can be expressed in terms of $E$ and $\nu$ as

$$
\epsilon_{i j}=\frac{1}{E}\left[\sigma_{i j}-\nu\left(\sigma_{k k} \delta_{i j}-\sigma_{i j}\right)\right]
$$

where $\delta_{i j}$ is the Kronecker delta. The idealized case of uniaxial strain, where strain is applied only in one direction (e.g. vertical) and where the lateral strains are suppressed, i.e. $\epsilon_{x x}=\epsilon_{y y}=0$, implies that

$$
\sigma_{x x}=\sigma_{y y}=\frac{\nu}{(1-\nu)} \sigma_{z z}
$$

which can be rearranged to calculate Poisson's ratio as

$$
\nu=\frac{\sigma_{x x}}{\left(\sigma_{z z}+\sigma_{x x}\right)}
$$

where $\nu\left(\sigma_{x x}\right)$ is equal to $\nu\left(\sigma_{y y}\right)$ if the material can be considered as isotropic and homogeneous. The constrained modulus $M$

$$
\frac{\sigma_{z z}}{\epsilon_{z z}}=M=E \frac{(1-\nu)}{(1+\nu)(1-2 \nu)}
$$

can be used to calculate $E$ when $\sigma_{z z}, \epsilon_{z z}$ and $\nu$ are known. The size of the RVE, and hence the size of the calculated subvolumes, was chosen according to $E$. However, a RVE for $E$ is not necessarily large enough to also hold the assumption of structural homogeneity for $\nu$. The structural variability in the horizontal plane $(x-y$ plane) affects $\nu$ more than $E$, and the volume size meeting the RVE criteria for $E$ does not necessarily meet the RVE criteria for $\nu$. Therefore, the resultant apparent stresses, $\sigma_{x x}$ and $\sigma_{y y}$ (for some snow samples these were not equal), were used to calculate two Poisson's ratios using Eqn (4):

$$
\nu\left(\sigma_{x x}\right)=\frac{\sigma_{x x}}{\left(\sigma_{z z}+\sigma_{x x}\right)}
$$

and

$$
\nu\left(\sigma_{y y}\right)=\frac{\sigma_{y y}}{\left(\sigma_{z z}+\sigma_{y y}\right)}
$$

Consequently two Young's moduli were calculated using Eqn (5): $E\left(\nu\left(\sigma_{x x}\right)\right)$ and $E\left(\nu\left(\sigma_{y y}\right)\right)$. For layer analysis an averaged Young's modulus

$$
\bar{E}=\frac{1}{2}\left[E\left(\nu\left(\sigma_{x x}\right)\right)+E\left(\nu\left(\sigma_{y y}\right)\right)\right]
$$

and an averaged Poisson's ratio

$$
\bar{\nu}=\frac{1}{2}\left(\nu_{x x}+\nu_{y y}\right)
$$

were used. For the sake of simplicity $\bar{E}$ and $\bar{\nu}$ are denoted as $E$ and $\nu$ hereafter. When the difference between $\nu\left(\sigma_{x x}\right)$ and $\nu\left(\sigma_{y y}\right)$ exceeded $50 \%$, the assumption of structural homogeneity was considered unacceptable, so $E$ and $\nu$ of these samples were excluded from further analysis.

\section{Relative change in microstructure between layers}

First, the subvolumes of all layers were used to calculate the relative change between geometric parameters and $E$ and $\nu$ between adjacent layers. The relative per cent change $\Delta$ of a parameter $x$ between two layers was calculated as

$$
\Delta x=\frac{x-x_{\mathrm{ref}}}{x_{\mathrm{ref}}} 100
$$

where the value of the overlying layer was the reference value $x_{\text {ref }}$. If the value $x$ of the underlying layer exceeds $x_{\text {ref }}$ the relative change is positive, otherwise it is negative.

Second, the difference between weak layers and their adjacent layers, i.e. layer above and below, was examined. Relative changes were calculated according to Eqn (10), but always with the weak layer as the reference layer $\left(x_{\text {ref }}=x_{\mathrm{WL}}\right.$, where $W L$ is weak layer).

\section{RESUITS}

\section{Snow characterization}

Twenty-two of the 32 cast layered samples contained an embedded weak layer. A sample consisting of a depth hoar layer lying under a thin melt/freeze crust, both buried under rounded snow, is shown as an example in Figure 1. Morphologically, the high resolution of $10 \mu \mathrm{m}$ revealed the presence of very thin layers with a vertical thickness $<2 \mathrm{~mm}$, which were not observable in the field. Very thin layers were also revealed by strong changes in the calculated parameters. These were sometimes not even morphologically visible in the 3-D visualization. The densities in the layers were $100-500 \mathrm{~kg} \mathrm{~m}^{-3}$, the SSAs $4-44 \mathrm{~m}^{-1}$ and the size of pores $0.17-0.94 \mathrm{~mm}$. The values of layers chosen for FE calculations (104 layers from 32 samples) are listed in the supplementary material at http://www.igsoc.org/hyperlink/ 13j220suppl.pdf.

\section{RVE for Young's modulus}

The RVE for a FE calculation of $E$ based on tomography data was determined to be at least $(7 \mathrm{~mm})^{3}$. Figure 2 shows the RVE results for three low-density samples $\left(\rho \leq 300 \mathrm{~kg} \mathrm{~m}^{-3}\right)$ : RG (volume: $300^{3}$ voxel, $(9 \mathrm{~mm})^{3}$, resolution $\left.0.030 \mathrm{~mm}\right)$, FC (volume: $350^{3}$ voxel, $(14 \mathrm{~mm})^{3}$, resolution $0.040 \mathrm{~mm}$ ) and $\mathrm{DH}$ (volume: $298^{3}$ voxel, $(10.7 \mathrm{~mm})^{3}$, resolution $\left.0.036 \mathrm{~mm}\right)$. Resolution was lower for snow with large structures to calculate a sufficiently large volume with a reasonable computing time. The RVE with a threshold of $20 \%$ variability was $\sim(1.5 \mathrm{~mm})^{3}$ for density, but $\sim(6.5 \mathrm{~mm})^{3}$ for the Young's modulus. The actual size of the RVE for $E$ depends in a rather complex way on snow type and density: the larger the grain size and the lower the density, the larger the RVE. The 104 cubic subvolumes cut out of the individual layers as shown in Figure 1, which were finally used as geometric input for the FE calculations, had a side length of $7-9 \mathrm{~mm}$, a resolution of $0.03-0.04 \mathrm{~mm}$ and consisted of up to $10^{6}$ elements.

\section{Young's modulus and Poisson's ratio}

The logarithmically transformed Young's modulus $E$ correlates well with density (Fig. 3). The range of $E$ is $0.3-$ $1000 \mathrm{MPa}$ for densities $100-500 \mathrm{~kg} \mathrm{~m}^{-3}$. The fit with an exponential equation for densities $\rho$ of $150-250 \mathrm{~kg} \mathrm{~m}^{-3}$ is

$$
E=0.0061 \exp (0.0396 \rho), \quad R^{2}=0.68
$$

and for densities $250-450 \mathrm{~kg} \mathrm{~m}^{-3}$ the fit is

$$
E=6.0457 \exp (0.011 \rho), \quad R^{2}=0.92
$$

Our results showed no obvious grouping depending on snow type, except for melt forms (the results of $E$ and $\nu$ for all subvolumes are listed in the supplementary material at http://www.igsoc.org/hyperlink/13j220suppl.pdf).

Poisson's ratio $\nu$, in contrast, shows no clear correlation with density (Fig. 4). However, snow types are rather clearly 

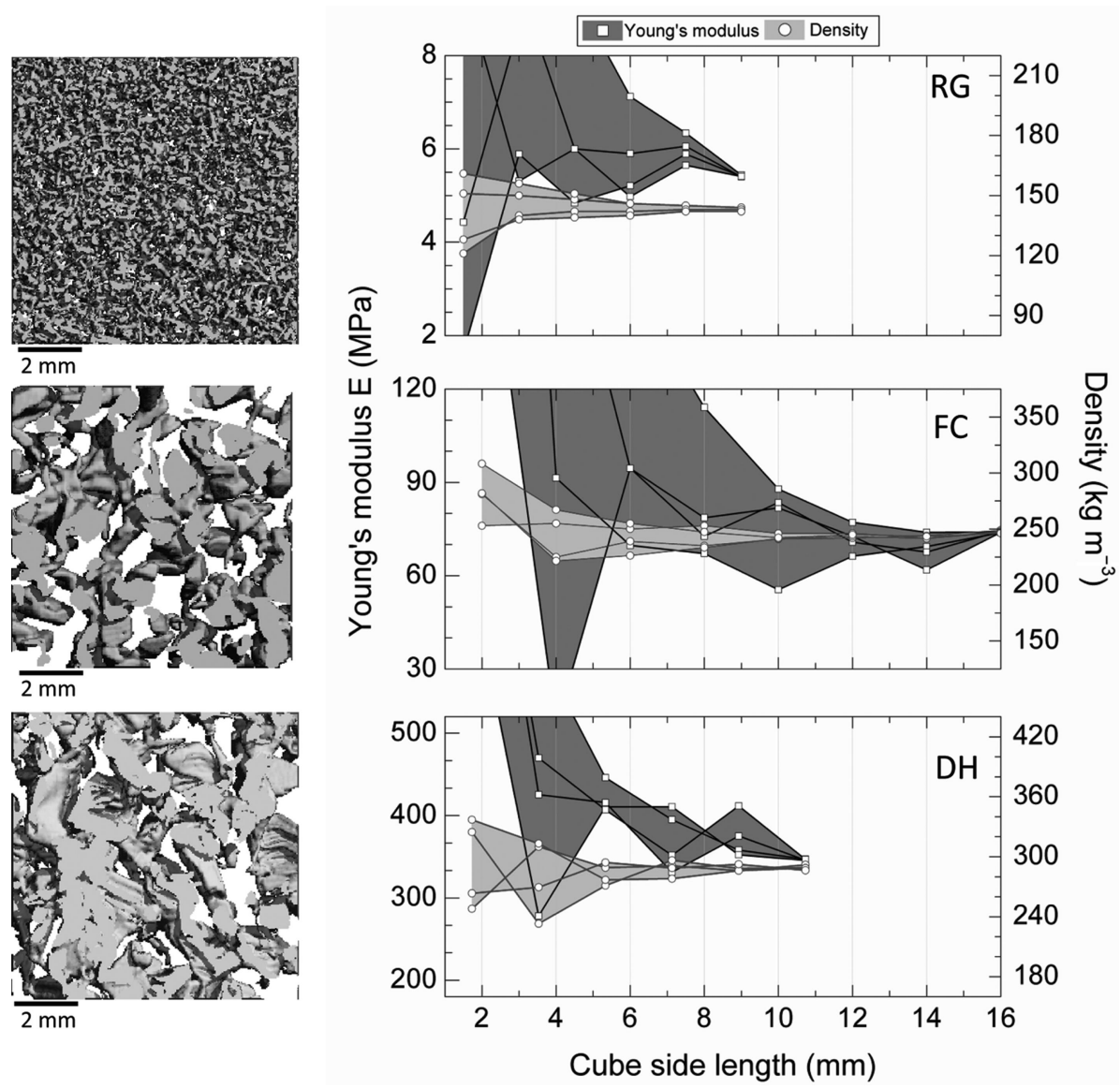

Fig. 2. RVE calculations for Young's modulus $E$ (left $y$-axis, dark-grey area) and density (right $y$-axis, light-grey area) vs cube side length. The RVE results for three samples are shown: rounded snow (RG, top), faceted snow (FC, middle) and depth hoar (DH, bottom). Each cube was calculated four times, starting from different corners to account for structural variability. The $\mu$-CT images on the left show a cut-out of $9 \mathrm{~mm} \times 9 \mathrm{~mm} \times 1.8 \mathrm{~mm}$ (resolution $0.03 \mathrm{~mm}$ ). A calculation of the Young's modulus requires a much larger RVE than a density calculation. In general, the larger the grain size and the lower the density, the larger the RVE.

grouped. RG shows an almost constant Poisson's ratio over the whole density range (mean $\nu_{\mathrm{RG}}(n=17) 0.171$; standard deviation \pm 0.026 ). In contrast, the Poisson's ratios of FC and $\mathrm{DH}$ were $<0.15$ and more scattered (mean $\nu_{\mathrm{FC}}(n=19)$ $0.130 \pm 0.040$; mean $\nu_{\mathrm{FC} / \mathrm{DH}}(n=6) 0.096 \pm 0.027$; mean $\left.\nu_{\mathrm{DH}}(n=6) 0.087 \pm 0.063\right)$.

\section{Relative microstructural change between layers}

The boundary between layers is defined by a distinct gradient in properties. The magnitude of this gradient can indicate a potential weak layer if both layers adjacent to the weak layer are considered. First the layers were not classified as weak or non-weak. Table 1 shows the results for 42 pairs. We found that the layer below was denser, showed reduced SSA, had fewer and thicker ice structures per millimetre, larger pores and, consequently, fewer structural connections. The upper and lower quartile of the median are not uniquely on one side of the median, so these observations are of more general trends. Large scatter is the rule for the layer-to-layer variability.

Second, the relative change from the perspective of the weak layer was calculated. All properties were therefore calculated with reference to the weak layer (Tables 2 and 3). We found that the weak layer and the two adjacent layers are sandwich-like: the layer above and layer below were, for most properties, closer to each other than to the weak layer.

Table 1. Relative change between layers, only non-disregarded data. Parameters are: $\rho$ : density; SSA: specific surface area; Tb.N: trabecular number; Th: ice thickness; Sp: pore thickness; Conn.D: connectivity density; $E$ : Young's modulus; $\nu$ : Poisson's ratio. $n$ is the number of pairs, and Q1 and Q3 are the first and the third quartile, respectively

\begin{tabular}{|c|c|c|c|c|c|c|c|c|}
\hline & $\Delta \rho$ & $\Delta \mathrm{SSA}$ & $\Delta$ Tb. $N$ & $\Delta \mathrm{Th}$ & $\Delta S p$ & $\Delta$ Conn.D & $\Delta E$ & $\Delta \nu$ \\
\hline & $\%$ & $\%$ & $\%$ & $\%$ & $\%$ & $\%$ & $\%$ & $\%$ \\
\hline$n$ & 41 & 41 & 41 & 41 & 41 & 41 & 41 & 31 \\
\hline Median & 6.37 & -2.01 & -6.24 & 2.04 & 4.76 & -19.07 & 32.25 & -4.75 \\
\hline Q1 & -25.51 & -12.48 & -27.57 & -2.67 & -17.51 & -70.76 & -70.03 & -14.82 \\
\hline Q3 & 27.48 & 2.73 & 18.20 & 14.26 & 56.90 & 48.81 & 283.64 & 13.27 \\
\hline Mean & 5.71 & -4.76 & -4.27 & 25.53 & 33.23 & 7.61 & 564.01 & 6.60 \\
\hline
\end{tabular}




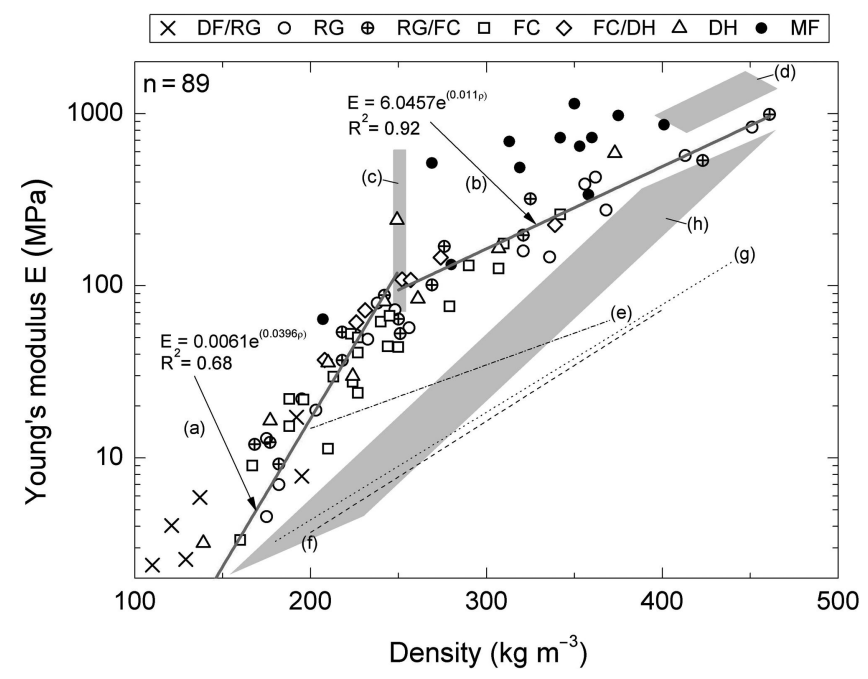

Fig. 3. Simulated Young's modulus $E$ vs density $\rho$ from this study compared with previous published data. Our own data were fitted once for low densities (100 $\leq \rho \leq 250 \mathrm{~kg} \mathrm{~m}^{-3}$; curve a) and once for densities $250-500 \mathrm{~kg} \mathrm{~m}^{-3}$ (curve b). Previously published data from FE simulations (Schneebeli, 2004, curve c; Srivastava and others, 2010, curve d) are similar in magnitude. In contrast, experimental results from laboratory measurements show lower values (Sigrist and others, 2006: curve e; Scapozza, 2004:, curve f; von Moos, as published by Stoffel and Bartelt, 2003: curve g; Shapiro and others, 1997: curve h). The symbols indicate grain shape according to Fierz and others (2009).

The most obvious difference was observed for density and elastic modulus. The weak layer was, on average, about half as dense as the layers above and below. Similarly, the elastic modulus was much higher (on average 20 times) in the layers above and below (Fig. 5). SSA was larger in the layer above compared with the weak layer and smaller in the

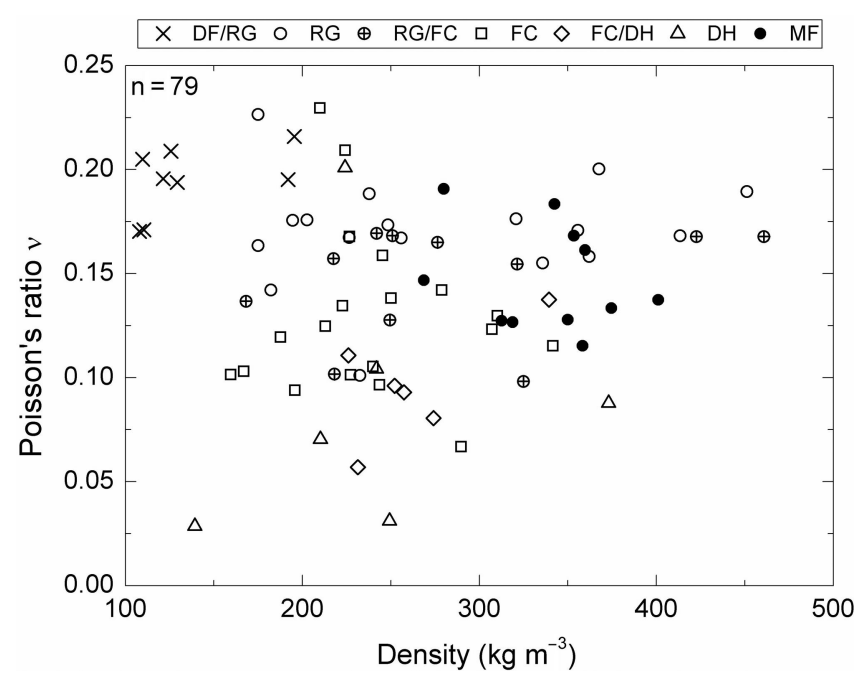

Fig. 4. Poisson's ratio $\nu$ vs density.

layer below. Ice thickness (Th) was inverse to SSA. The change in the number of ice structures (Tb.N) was positive for both the layers above and below, and consequently the pore size (Sp) for both was negative. The change in $\nu$ was positive for the layer above, and negative for the layer below, with $\nu$ for weak layers not necessarily lower than for the layers above and below (Fig. 6).

\section{DISCUSSION}

Three-dimensional data for snow obtained by X-ray measurements and image processing make it possible to measure the snow microstructure objectively and to perform geometrybased numerical simulations. We used these methods to investigate snow stratigraphy at a scale of $10^{-2}-10^{-4} \mathrm{~m}$, with

Table 2. Relative change between the weak layer and the layer above. Parameters are the same as in Table 1

\begin{tabular}{|c|c|c|c|c|c|c|c|c|}
\hline & $\Delta \rho$ & $\Delta \mathrm{SSA}$ & $\Delta$ Tb. $N$ & $\Delta \mathrm{Th}$ & $\Delta \mathrm{Sp}$ & $\Delta$ Conn.D & $\Delta E$ & $\Delta \nu$ \\
\hline & $\%$ & $\%$ & $\%$ & $\%$ & $\%$ & $\%$ & $\%$ & $\%$ \\
\hline$n$ & 11 & 11 & 11 & 11 & 11 & 11 & 11 & 8 \\
\hline Median & 62.60 & 6.11 & 68.13 & -5.76 & -50.45 & 231.09 & 958.66 & 23.53 \\
\hline Q3 & 98.93 & 11.23 & 119.32 & 56.41 & -31.85 & 454.25 & 3518.64 & 42.46 \\
\hline Mean & 71.70 & -1.83 & 63.73 & 33.67 & -34.27 & 265.25 & 2707.42 & 32.93 \\
\hline
\end{tabular}

Table 3. Relative change between the weak layer and the layer below. Parameters are the same as in Table 1

\begin{tabular}{|c|c|c|c|c|c|c|c|c|}
\hline & $\Delta \rho$ & $\Delta \mathrm{SSA}$ & $\Delta$ Tb.N & $\Delta \mathrm{Th}$ & $\Delta S p$ & $\Delta$ Conn.D & $\Delta E$ & $\Delta \nu$ \\
\hline & $\%$ & $\%$ & $\%$ & $\%$ & $\%$ & $\%$ & $\%$ & $\%$ \\
\hline$n$ & 8 & 8 & 8 & 8 & 8 & 8 & 8 & 7 \\
\hline Median & 43.28 & -4.25 & 19.52 & 4.76 & -26.27 & 39.14 & 606.58 & -15.38 \\
\hline Q3 & 72.44 & 2.02 & 42.39 & 49.33 & -6.78 & 88.77 & 1879.60 & -12.55 \\
\hline Mean & 56.78 & -18.87 & 24.15 & 65.13 & -11.52 & 69.00 & 1964.73 & 26.17 \\
\hline
\end{tabular}




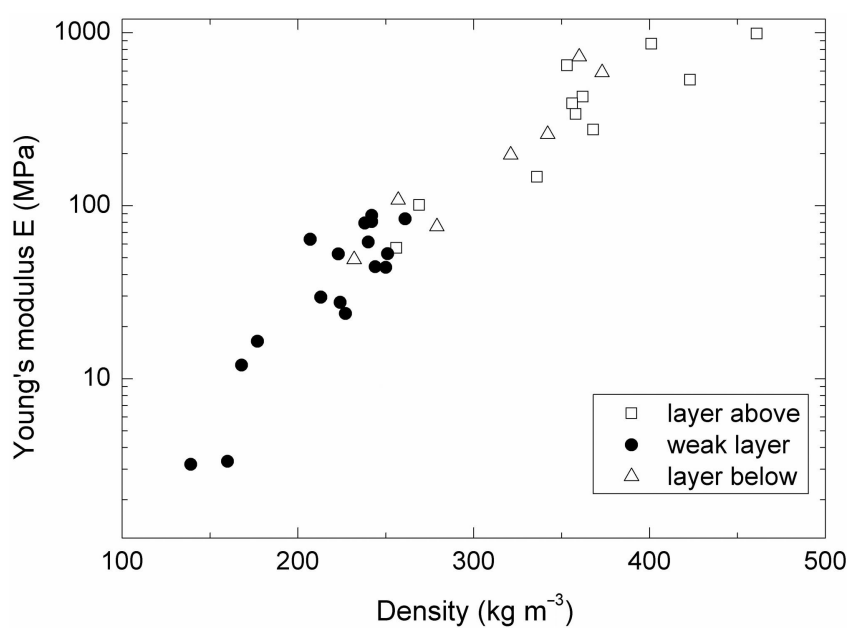

Fig. 5. Young's modulus $E$ vs density for subsamples within weak layers $(n=17)$, the layer above the weak layer $(n=11)$ and the layer below the weak layer $(n=7)$. If $E$ is considered as a measure of stiffness, the weak layer is much less stiff than its adjacent layers.

a focus on the microstructure of weak layers. The geometrybased simulations helped to differentiate the layers in a sample, not only by the change in morphology or measured microstructure, but also by changes in mechanical properties.

\section{Computational aspects}

Previous studies have demonstrated the importance of the RVE for a FE calculation of physical properties (e.g. as discussed in a mechanical context by Srivastava and others (2010) and shown in studies of thermal properties of snow by Kaempfer and others (2005) and Calonne and others (2011)). Here we calculated the RVE with respect to Young's modulus for low-density snow. The required minimal cube side length for a sample depends on the density and the snow type. The larger the structural size and the lower the density, the larger the RVE.

For the FE calculations, the extraction of subvolumes of the layers - for this purpose defined as regions with constant microstructure (e.g. SSA or density) and with an apparently constant grain shape - was done with respect to the calculated RVE, resulting in a cube side length of at least $7 \mathrm{~mm}$. The shape of the volume has to be cubic, based on the following considerations: $7 \mathrm{~mm}$ in the vertical direction, $z$, includes enough structure for even large grains (e.g. DH). Horizontal $(x-y$ plane) spreading is necessary to obtain structural homogeneity in this plane, important for Poisson's ratio. Similar considerations were reported by Kaempfer and others (2005) in a thermal context. The requirement to meet the RVE size had the drawback that the properties of layers thinner than $7 \mathrm{~mm}$ were not calculated, because their resulting elastic constants were biased.

Simulating only one load case (uniaxial strain in the vertical direction), we did not calculate the full elasticity tensor and hence a true vertical Young's modulus, $E_{z z}$. Furthermore $E$ and $\nu$ were calculated from the $\mathrm{FE}$ results under the assumption of isotropy and structural homogeneity of the cubic subvolumes. This introduces bias in our values. Nevertheless, for the purpose of studying layering, we consider our calculations to be sufficient. The assumption of isotropy and homogeneity is also made in the analysis of laboratory experiments and for layer properties in fracturemechanical models of slab avalanche release.

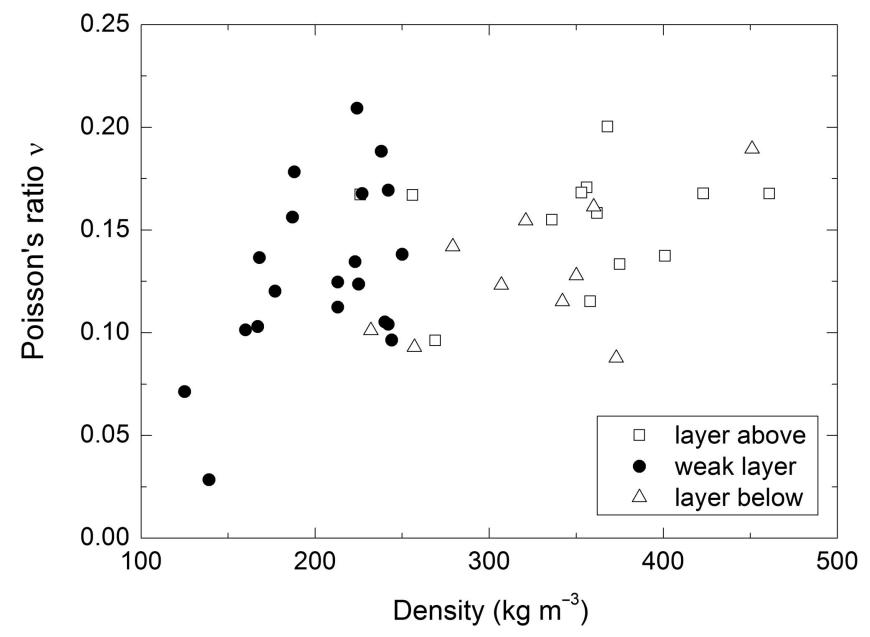

Fig. 6. Poisson's ratio $\nu$ vs density for subsamples within weak layers $(n=20)$, the layer above the weak layer $(n=13)$ and the layer below the weak layer $(n=10)$.

\section{Young's modulus and Poisson's ratio}

The log-transformed calculated Young's moduli correlated well with density. We compared our data with previously published values from laboratory experiments (Shapiro and others, 1997; Stoffel and Bartelt, 2003; Scapozza, 2004; Sigrist and others, 2006) and from FE simulations with real 3-D snow microstructures (Schneebeli, 2004; Srivastava and others, 2010) (Fig. 3). Data from FE calculations generally give higher values than data obtained experimentally (this was also noted by Srivastava and others, 2010). Apart from the large variety of measurement methods, we interpret the lower values of $E$ obtained from the experimental data as an effect of the low strain rates used for determining the elastic modulus, which introduce viscous effects.

For densities $<250 \mathrm{~kg} \mathrm{~m}^{-3}$ the slope is different to that for higher densities (Fig. 3), and the correlation is lower ( $R^{2}=0.68 \mathrm{cf}$. 0.92 for higher densities). We suggest that the lower correlation of $E$ with lower densities is due to a greater influence of microstructure. Structural dead ends that do not contribute to the stress distribution have a larger impact in snow samples with low densities and introduce scatter.

Although our calculated elastic constants were primarily used to identify layers, we consider it an interesting result that no dependence on morphological snow type was found for $E$ (Fig. 3). To be more precise, no range for $E$ can be assigned to a given snow type. As a consequence, it cannot be generalized that a low Young's modulus per se indicates weak snow: Young's moduli of the weak layers were only low relative to the values in their adjacent layers (Fig. 5). To use $E$ as an indicator for weak layers, the 'sandwich', i.e. both the weak layer and the two adjacent layers, must be considered.

Microstructure could have more influence on Poisson's ratio. If Poisson's ratio is close to zero, the material is more compressible and more resistant to transverse contraction or expansion. This is associated with materials having stiff structures in the direction normal to the loading (Greaves, 2011). Figure 4 shows that $\mathrm{DH}$ and $\mathrm{DH}$ mixed with facets have the lowest Poisson's ratio. This would support the picture of vertically oriented load-bearing chains within $\mathrm{DH}$ which expand little in the horizontal direction. We note, however, that Poisson's ratio within the sandwich does not distinguish the weak layers as $E$ does. Figure 6 shows that 
weak layers can consist of many different structures, and not only of cup crystals and facets.

\section{Layering at the microstructural scale}

Layer transitions in our high-resolution $\mu$-CT samples have a natural roughness given by the size of the microstructure. The high resolution also revealed layers only a few millimetres thick, which were often not observable in the field. Assigning the layers within the $\mu$-CT sample to the layers found in the field was challenging. When the weak layer or the adjacent layers (defined as such in the field) were not clearly distinguishable in the corresponding $\mu$-CT sample, the subvolume was not considered in the context of examining structural changes around a weak layer. We found that microstructural parameters from $\mu$-CT measurements distinguished an a priori known weak layer from nonweak layers, not by absolute values but by the magnitude of their relative change. It is important to note that the relative change has to be calculated for the sandwich, i.e. layerabove-weak-layer-layer-below. By considering only two layers, a large change could also be due to a crust and is not necessarily due to a weak layer. More generally, the difference between a stratigraphic sequence without and with an embedded weak layer is the magnitude of the relative microstructural change over three adjacent layers.

\section{CONCLUSION}

The 3-D microstructure of 32 layered snow samples, cast in situ, with 22 embedded weak layers, was reconstructed with $\mathrm{X}$-ray microcomputer tomography. The high-resolution data allowed objective quantification of structural changes at a scale of $10^{-2}-10^{-4} \mathrm{~m}$. Layers were distinguished by morphology, microstructural parameters and the elastic constants, Young's modulus $E$ and Poisson's ratio $\nu$. The two latter were calculated from CT-data-based FE calculations. This required a definition of a RVE with respect to Young's modulus, as a RVE determined with respect to density would be too small to capture the representative elastic bulk properties. The relative vertical change in microstructure and elastic parameters was calculated for each sample. Layers were best distinguished through relative differences, but, except for Young's modulus, no parameter consistently accounted for a structural change. Elastic properties are characteristic for a certain microstructure and $E$ and $\nu$ are closely linked to the fracture properties of the snow. Mechanical properties are therefore a much better parameter to differentiate weak layers than purely structural properties. The comparison between layering in general and layering with a weak layer showed that the weak layer is most clearly distinguished by a strong relative change in microstructure when compared to both adjacent layers, and not by a single value.

\section{ACKNOWLEDGEMENTS}

M. Matzl processed the cast samples and evaluated the CT scans. M. Matzl, M. Proksch and J. Singer helped to sample the fragile snow. We thank Scanco Medical for support.

\section{REFERENCES}

Bellaire S, Pielmeier C, Schneebeli $M$ and Schweizer J (2009) Stability algorithm for snow micro-penetrometer measurements. J. Glaciol., 55(193), 805-813 (doi: 10.3189/ 002214309790152582)

Calonne N, Flin F, Morin S, Lesaffre B, Rolland du Roscoat S and Geindreau C (2011) Numerical and experimental investigations of the effective thermal conductivity of snow. Geophys. Res. Lett., 38(23), L23501 (doi: 10.1029/2011GL049234)

Coléou C, Lesaffre B, Brzoska JB, Ludwig W and Boller E (2001) Three-dimensional snow images by $\mathrm{X}$-ray microtomography. Ann. Glaciol., 32, 75-81 (doi: 10.3189/172756401781819418)

Fierz C and 8 others (2009) The international classification for seasonal snow on the ground. (IHP Technical Documents in Hydrology 83) UNESCO-International Hydrological Programme, Paris

Föhn PMB, Camponovo C and Krüsi G (1998) Mechanical and structural properties of weak snow layers measured in situ. Ann. Glaciol., 26, 1-6

Greaves GN, Greer AL, Lakes RS and Rouxel T (2011) Poisson's ratio and modern materials. Nature Mater., 10(11), 823-837

Heggli $M$ and 7 others (2011) Measuring snow in 3-D using X-ray tomography: assessment of visualization techniques. Ann. Glaciol., 52(58), 231-236 (doi: 10.3189/172756411797252202)

Heierli J, Gumbsch P and Zaiser M (2008) Anticrack nucleation as triggering mechanism for snow slab avalanches. Science, 321(5886), 240-243 (doi: 10.1126/science.1153948)

Hildebrand T and Rüesegger P (1997) A new method for the modelindependent assessment of thickness in three-dimensional images. J. Microsc., 185(1), 67-75 (doi: 10.1046/j.13652818.1997.1340694.x)

Johnson JB and Schneebeli M (1999) Characterizing the microstructural and micromechanical properties of snow. Cold Reg. Sci. Technol., 30(1-3), 91-100 (doi: 10.1016/S0165-232X(99) 00013-0)

Kaempfer TU, Schneebeli M and Sokratov SA (2005) A microstructural approach to model heat transfer in snow. Geophys. Res. Lett., 32(21), L21503 (doi: 10.1029/2005GL023873)

Kronholm K, Schneebeli M and Schweizer J (2004) Spatial variability of micropenetration resistance in snow layers on a small slope. Ann. Glaciol., 38, 202-208

Kry PR (1975) The relationship between the visco-elastic and structural properties of fine-grained snow. J. Glaciol., 14(72), 479-500

Löwe H and Van Herwijnen A (2012) A Poisson shot noise model for micro-penetration of snow. Cold Reg. Sci. Technol., 70, 62-70 (doi: 10.1016/j.coldregions.2011.09.001)

Löwe H, Riche F and Schneebeli M (2013) A general treatment of snow microstructure exemplified by an improved relation for thermal conductivity. Cryosphere, 7(5), 1473-1480 (doi: 10.5194/tc-7-1473-2013)

Lutz E, Birkeland KW and Marshall HP (2009) Quantifying changes in weak layer microstructure associated with artificial load changes. Cold Reg. Sci. Technol., 59(2-3), 202-209 (doi: 10.1016/j.coldregions.2009.04.003)

Marshall HP and Johnson JB (2009) Accurate inversion of highresolution snow penetrometer signals for microstructural and micromechanical properties. J. Geophys. Res., 114(F4), F04016 (doi: 10.1029/2009JF001269)

McClung DM (1981) Fracture mechanical model of dry slab avalanche release. J. Geophys. Res., 86(B11), 10783-10790 (doi: 10.1029/JB086iB11p10783)

Narita $\mathrm{H}$ (1980) Mechanical behaviour and structure of snow under uniaxial tensile stress. J. Glaciol., 26(94), 275-282

Odgaard A and Gundersen HJG (1993) Quantification of connectivity in cancellous bone, with special emphasis on 3-D reconstructions. Bone, 14(2), 173-182 (doi: 10.1016/87563282(93)90245-6)

Petrovic JJ (2003) Review mechanical properties of ice and snow. J. Mater. Sci., 38(1), 1-6 (doi: 10.1023/A:1021134128038)

Pielmeier C and Schweizer J (2007) Snowpack stability information derived from the SnowMicroPen signal. Cold Reg. Sci. Technol., 47(1-2), 102-107 (doi: 10.1016/j.coldregions.2006.08.013) 
Pieritz RA, Brzoska JB, Flin F, Lesaffre B and Coléou C (2004) From snow $\mathrm{X}$-ray microtomograph raw volume data to micromechanics modeling: first results. Ann. Glaciol., 38, 52-58 (doi: 10.3189/172756404781815176)

Scapozza C (2004) Entwicklung eines dichte- und temperaturabhängigen Stoffgesetzes zur Beschreibung des visko-elastischen Verhaltens von Schnee. (PhD thesis, ETH Zürich)

Schneebeli M (2002) The importance of the microstructure of snow in nature and engineering. In Brebbia CA ed. Design and nature III: comparing design in nature with science and engineering. WIT Press, Southampton, 87-93

Schneebeli M (2004) Numerical simulation of elastic stress in the microstructure of snow. Ann. Glaciol., 38, 339-342 (doi: 10.3189/172756404781815284)

Schneebeli M, Pielmeier C and Johnson JB (1999) Measuring snow microstructure and hardness using a high resolution penetrometer. Cold Reg. Sci. Technol., 30(1-3), 101-114 (doi: 10.1016/ S0165-232X(99)00030-0)

Schweizer J and Wiesinger T (2001) Snow profile interpretation for stability evaluation. Cold Reg. Sci. Technol., 33(2-3), 179-188 (doi: 10.1016/S0165-232X(01)00036-2)

Schweizer J, Jamieson JB and Schneebeli M (2003) Snow avalanche formation. Rev. Geophys., 41(4), 1016 (doi: 10.1029/ 2002RG000123)

Shapiro LH, Johnson JB, Sturm M and Blaisdell GL (1997) Snow mechanics: review of the state of knowledge and applications. CRREL Rep. 97-3

Sigrist C, Schweizer J, Schindler H-J and Dual J (2006) The energy release rate of mode II fractures in layered snow samples. Int. J. Frac., 139(3-4), 461-475 (doi: 10.1007/s10704-006-6580-9)
Srivastava PK, Mahajan P, Satyawali PK and Kumar V (2010) Observation of temperature gradient metamorphism in snow by $\mathrm{X}$-ray computed microtomography: measurement of microstructure parameters and simulation of linear elastic properties. Ann. Glaciol., 51(54), 73-82 (doi: 10.3189/172756410791386571)

Stoffel M and Bartelt P (2003) Modelling snow slab release using a temperature-dependent viscoelastic finite element model with weak layers. Surv. Geophys., 24(5-6), 417-430 (doi: 10.1023/ B:GEOP.0000006074.56474.43)

Theile T, Löwe H, Theile TC and Schneebeli M (2011) Simulating creep of snow based on microstructure and the anisotropic deformation of ice. Acta Mater., 59(18), 7104-7113 (doi: 10.1016/j.actamat.2011.07.065)

Van Herwijnen A, Bellaire S and Schweizer J (2009) Comparison of micro-structural snowpack parameters derived from penetration resistance measurements with fracture character observations from compression tests. Cold Reg. Sci. Technol., 59(2-3), 193-201 (doi: 10.1016/j.coldregions.2009.06.006 )

Van Rietbergen B, Weinans H, Huiskes R and Odgaard A (1995) A new method to determine trabecular bone elastic properties and loading using micromechanical finite-element models. J. Biomech., 28(1), 69-81 (doi: 10.1016/0021-9290(95)80008-5)

Van Rietbergen B, Odgaard A, Kabel J and Huiskes R (1996) Direct mechanics assessment of elastic symmetries and properties of trabecular bone architecture. J. Biomech., 29(12), 1653-1657 (doi: 10.1016/S0021-9290(96)80021-2)

Winkler K and Schweizer J (2009) Comparison of snow stability tests: extended column test, rutschblock test and compression test. Cold Reg. Sci. Technol., 59(2-3), 217-226 (doi: 10.1016/ j.coldregions.2009.05.003)

MS received 29 November 2013 and accepted in revised form 22 April 2014 